\title{
Correspondence
}

Korean J Ophthalmol 2021;35(4):325-327

https://doi.org/10.3341/kjo.2021.0030

\section{Surgical Tips for Approaching to Superior Ophthalmic Vein in Patients with a Carotid Cavernous Fistula}

\section{Dear Editor,}

I am writing to present a surgical tip for approaching to superior ophthalmic vein (SOV) of indirect carotid cavernous fistula. The SOV approach was performed by a single oculoplastic surgeon (PJY) in the following protocol. Under general anesthesia, a radiopaque angio-catheter fragment (ACF) of 10-mm length (A\&A MD, Seongnam, Korea) was placed under the sub-brow area. The relative location of the orbital rim, SOV, and ACF was evaluated through angiography (Fig. 1A), and 10- to $15-\mathrm{mm}$ incision was made on the same line where the antero-most part of the SOV. It was the shortest plane from SOV. The dissection was continued to just beneath the superior orbital rim, and orbital fat was exposed and gently dissected using a cotton applicator. The SOV is easily identified at the same surgical plane where the ACF was placed. After identifying the SOV using doppler ultrasound, to straighten and control the SOV, the silk thread was put under the SOV using a muscle hook with a hole. It allows to easily approach to the narrow, deep space and it helps to dissect the surrounding soft tissues around the SOV (Fig. 1B). Next, the vessel was punctured with an 18-gauge angio-catheter. After embolization, the SOV was ligated with the pre-positioned silk sutures. During the operation, hemostasis was achieved by the cottonoid absorbent in a solution of 1 : 100,000 of epinephrine and normal saline. The incision was sutured with 6-0 silk sutures.

Received: March 19, 2021 Final revision: April 21, 2021

Accepted: April 26, 2021
Two female patients, aged 68 (case 1) and 51 (case 2), underwent the SOV approach using an ACF and muscle hook with a hole as described above in detail. The follow-up period was 6 months. The primary diagnosis in case 1 was right indirect carotid cavernous fistula with inferior petrosal sinus obstruction (Fig. 1C). Visual acuity was 0.1 right and 0.4 left, and the intraocular pressure was $27 \mathrm{mmHg}$ right and $18 \mathrm{mmHg}$ left. She showed proptosis and full limitation of extraocular movement on abduction of her right eye. In case 2 , right indirect carotid cavernous fistula with a dilated superficial middle cerebral vein and prominent posterior flow and cortical venous reflux was observed (Fig. 1D).

The most direct and usual route to the cavernous sinus is the inferior petrosal sinus due to its direct connection to the internal jugular vein [1]. However, both patients have their conventional pathway of embolization, the ipsilateral and the contralateral inferior petrosal sinus were obstructed. Therefore, the authors attempted an ipsilateral SOV approach as described above and basically based on the method as we reported, previously [2].

Some variations in the anatomy and the dimensions of the orbital veins make circumstances difficult [3,4]. Moreover, if the veins are recently developed, shunts would be more prone to perforation and difficult to control [5].

Using a radiopaque ACF during operation allows prompt intraoperative identification of the SOV. It helps us to presume the location of SOV even in case the vessels are obstructed with thrombosis or anatomic variation of the SOV. It can be placed on the skin or into the dissected soft tissue, and an angiogram is performed through the transfemoral vein let the relative location of the SOV and the angio-catheter be easily identified. Moreover, it informs us which direction the catheter should be inserted to approach to the cavernous sinus. There may be a worrisome opinion of an unnecessary additional exposure of radiation to the patients, but this method rather reduces the excessive expo- 

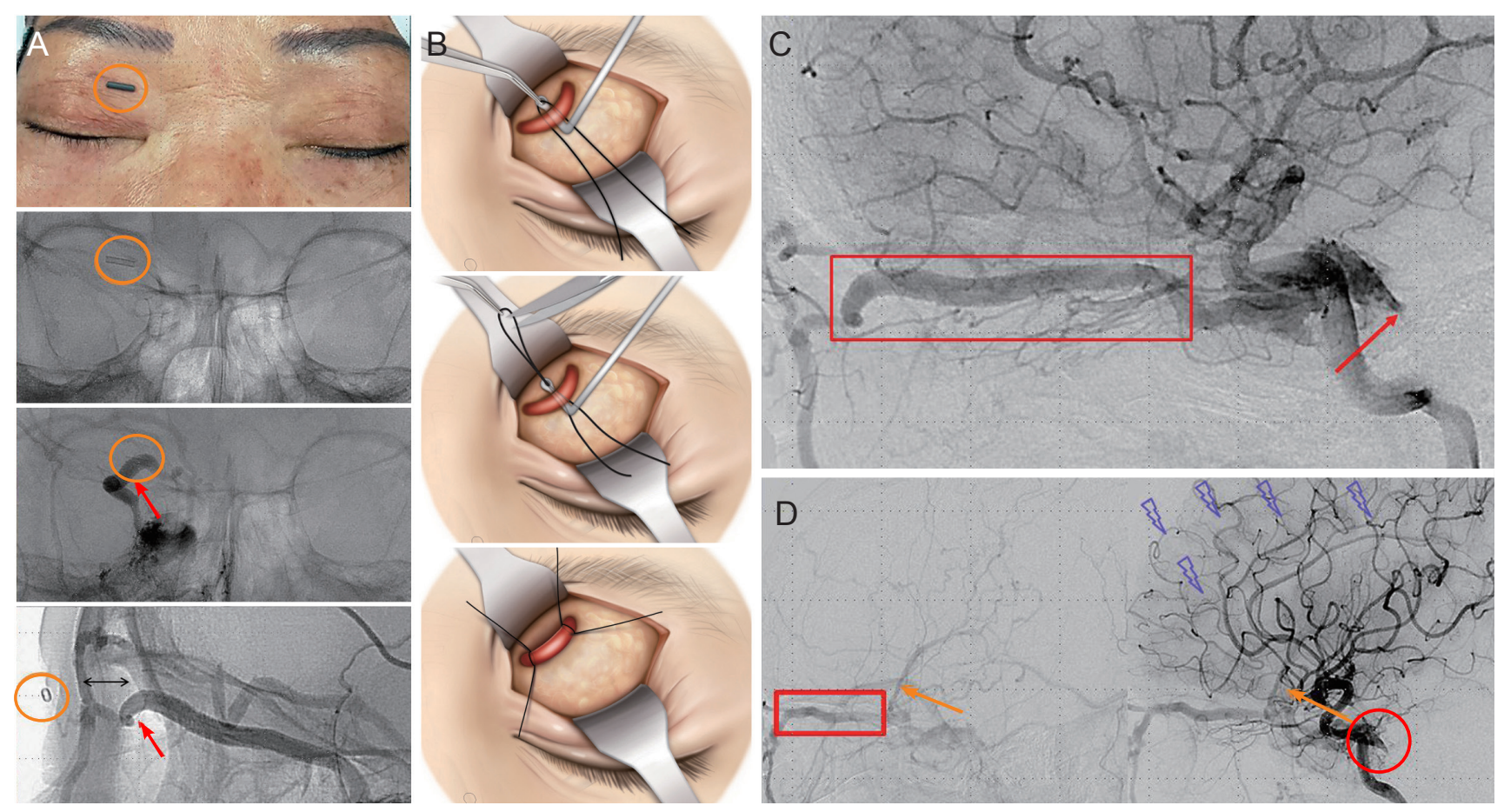

Fig. 1. Photograph, angiographic findings and schematic illustrations of surgical technique to approach superior ophthalmic vein. (A) The images of technique to evaluate the location of the superior ophthalmic vein (SOV) (red arrows). The radiopaque angio-catheter fragment (orange circles), was placed on the skin or dissected connective tissue and angiography was performed. The relative location of the angio-catheter orbital rim, and the SOV was identified. Minimal incision for direct approach can be performed and the SOV was successfully secured. (B) Schematic images of approaching the deep positioned SOV using a muscle hook with a hole. Put a 4-0 silk thread into the hole of a muscle hook and insert the muscle hook just beneath the SOV. Additional blunt dissection can be performed with the muscle hook. Pull out the silk thread using a non-tooth forceps and cut the middle part of the silk thread. Then, locate the two strands proximally and distally. Tie carefully at each side of the SOV for easy manipulation and hemostasis in case of vascular rupture. (C) Angiography via bilateral internal carotid artery shows severely dilated SOV (red box) and obstruction of the inferior petrosal sinus (red arrow). (D) Angiography shows dilated SOV (red box) and superficial middle cerebral vein (orange arrows) drained by the tentorial sinus. The entrance site of the inferior petrosal sinus was obstructed (red circle) and cortical venous reflux was observed (fragmented purple arrows). Informed consent for publishing patient photographs was obtained.

sure of radiation by approaching SOV easily and quickly.

Previous reports recommend to use a mosquito forceps to put the silk threads under the SOV [5], but we found it difficult to reach under the SOV using a mosquito forceps due to the narrow, deep, and small incision site. Using a muscle hook with a hole and placing the silk thread into the hole, allows surgeons to approach easily and safely to posterior part of the SOV. Furthermore, the connective tissues and adjacent structures can be easily dissected with the blunt tip of the muscle hook; it is placed under the SOV and the silk threads are pulled out from the hole of the muscle hook, cut into two threads, allowing a safe secure of the SOV before and end of the surgery.
Jungyul Park

Department of Ophthalmology, Biomedical Research Institute, Pusan National University Hospital, Pusan National University School of Medicine, Busan, Korea

\section{Jae-Il Lee}

Department of Neurosurgery, Pusan National University Hospital, Busan, Korea

\section{Hee-Young Choi}

Department of Ophthalmology, Biomedical Research Institute, Pusan National University Hospital, Pusan National University School of Medicine, Busan, Korea

E-mail:hychoi@pusan.ac.kr 


\section{Conflict of Interest}

No potential conflict of interest relevant to this article was reported.

\section{Acknowledgements}

This work was supported by clinical research grant from Pusan National University Hospital in 2021.

\section{References}

1. Park SH, Park KS, Kang DH, et al. Stereotactic radiosurgery for dural carotid cavernous sinus fistulas. World Neurosurg 2017;106:836-43.
2. Park J, Lee JI, Jeon H, Choi HY. Direct approach to thrombosed superior ophthalmic vein of recalcitrant indirect carotid cavernous fistula in thrombocythemia failed with multiple conventional embolization treatment. Int J Ophthalmol 2020;13:687-92.

3. Uflacker R, Lima S, Ribas GC, Piske RL. Carotid-cavernous fistulas: embolization through the superior ophthalmic vein approach. Radiology 1986;159:175-9.

4. Lim NK, Kim JK, Kim EJ, Yoo JM. Coil embolization via a superior ophthalmic vein approach of carotid cavernous sinus fistula. J Korean Ophthalmol Soc 2006;47:1141-8.

5. Leibovitch I, Modjtahedi S, Duckwiler GR, Goldberg RA. Lessons learned from difficult or unsuccessful cannulations of the superior ophthalmic vein in the treatment of cavernous sinus dural fistulas. Ophthalmology 2006;113: 1220-6. 